\title{
Predicting urinary incontinence after surgery for pelvic organ prolapse
}

\author{
John E. Jelovsek
}

\begin{abstract}
Purpose of review
Many women choosing to have surgery for pelvic organ prolapse also choose to undergo continence surgery. This review focuses on available evidence that clinicians may use to counsel patients when choosing whether to perform continence surgery and how predictive analytic tools improve this decision-making process.

\section{Recent findings}

Midurethral sling, Burch cystourethropexy and bladder neck sling are highly effective for the surgical treatment of stress urinary incontinence. Trials demonstrate that continence surgery may be routinely performed to reduce the risk of postoperative incontinence in women undergoing surgery for pelvic organ prolapse with or without preoperative stress urinary incontinence. Although these procedures are effective and well tolerated on average, media concerns, regulatory warnings and litigation reinforce the need for a balanced discussion regarding efficacy and potential adverse events directed at the individual patient during the preoperative visit. Advances in predictive analytics allow surgeons to quantitate individual risk using algorithms that tailor estimates for the individual patient and facilitate shared understanding of risks and benefits. These models are less prone to cognitive biases and frequently outperform experienced clinicians.
\end{abstract}

\section{Summary}

This review discusses how predictive analytic tools can be used to improve decisions about continence surgery in the woman planning to undergo prolapse surgery.

Keywords

adverse events, pelvic organ prolapse, prediction models, urinary incontinence

\section{INTRODUCTION}

Urinary incontinence will develop after prolapse repair in approximately one-quarter of patients with advanced pelvic organ prolapse who remain continent despite significant loss of anterior vaginal and pelvic organ support [1]. Many women with advanced pelvic organ prolapse who choose to undergo surgical management also choose to undergo continence surgery to prevent new-onset urinary incontinence. Midurethral sling, Burch cystourethropexy and bladder neck sling are highly effective treatment options for stress urinary incontinence in these situations [1]. In addition, when women complain of preoperative incontinence, multiple trials demonstrate that over $80 \%$ of these women are cured or have a significant improvement in their symptoms up to 5 years after placement of midurethral slings [2].

Recent regulatory statements and litigation have heightened the public's awareness and scrutiny of these procedures. This has reinforced the need for surgeons to improve their counseling prior to surgery and ensure that they provide an evidence-based, balanced discussion regarding efficacy and adverse event rates for the individual patient. This need presents several unique, and often, time-consuming challenges. The surgeon should convey the most accurate information regarding risks and benefits available and allow the patient to use this information during shared decision-making.

Obstetrics, Gynecology \& Women's Health Institute, Cleveland Clinic, Cleveland, Ohio, United States

Correspondence to John E. Jelovsek, MD, MMEd, Obstetrics, Gynecology \& Women's Health Institute, Cleveland Clinic, 9500 Euclid Avenue A81, Cleveland, OH 44195, United States. Tel: +1 216444 2488; e-mail: jelovsj@ccf.org

Curr Opin Obstet Gynecol 2016, 28:000-000

DOI:10.1097/GCO.0000000000000308 


\section{KEY POINTS}

- Randomized continence trials were designed to make relative comparisons of two or more treatments using odds ratios, hazard ratios or other measures of relative effects or harms.

- Surgical trials provide relative effects of the average patient in each treatment arm and do not emphasize individual risk such that low-risk patients have a lower absolute risk and higher risk patients have a higher absolute risk from the average patient in each treatment arm.

- Accurate predictive analytic tools in the form of clinical prediction models exist that allow a surgeon to accurately counsel a patient by providing an absolute risk for the individual given her characteristics.

- Clinical prediction models frequently outperform clinicians or other treatment decision protocols. When predicting risk of de novo stress urinary incontinence after surgery for pelvic organ prolapse, model predictions outperform a stress test and a group of experienced surgeon's predictions. This model is available in an online calculator.

The current review uses examples of women with and without preoperative stress urinary incontinence who are planning to undergo surgery for pelvic organ prolapse and who are considering a concomitant continence procedure. The examples will illustrate common strategies surgeons consider when informing the patient during this decisionmaking process. The examples also illustrate how predictive analytic tools may inform the decision process in women with individual risks different from an average group of women.

\section{DE NOVO STRESS URINARY INCONTINENCE}

Case 1: The patient is a 78-year-old para 2 woman who is planning to undergo a vaginal hysterectomy, uterosacral vaginal vault suspension, cystocele repair and cystoscopy for bothersome pelvic organ prolapse. She does not report any urinary incontinence but does report intermittent difficulty emptying her bladder. Her past medical history is significant for hypertension and hypercholesterolemia but no additional medical problems. Her past surgical history is only significant for cholecystectomy. On physical exam, her BMI is $28 \mathrm{~kg} / \mathrm{m}^{2}$, and abdomen has right upper quadrant scar. On pelvic exam, her pelvic organ prolapse quantification system (POPQ) points are $\mathrm{Ba}+2, \mathrm{C} 0$ and $\mathrm{Bp}-2$. An office prolapse reduction stress test is performed, and leaking is visualized during cough.
She has decided to pursue surgical management of her prolapse, and the surgeon must now discuss options with her for managing the risk of new-onset stress urinary incontinence.

Case 2: The patient is a 52-year-old para 3 woman who is planning to undergo a vaginal hysterectomy, uterosacral vaginal vault suspension, cystocele repair and cystoscopy for bothersome pelvic organ prolapse. She reports some occasional leaking with a feeling of urgency. Her past medical history is significant for oral medication controlled diabetes mellitus. On physical exam, her BMI is $30 \mathrm{~kg} / \mathrm{m}^{2}$, and on pelvic exam her POPQ points are $\mathrm{Ba}+2, \mathrm{C} 0$ and $\mathrm{Bp}-2$. An office prolapse reduction stress test is performed, and leaking is not visualized. She has decided to pursue surgical management of her prolapse, and the surgeon must now discuss options with her for managing the risk of new-onset stress urinary incontinence.

The two cases illustrate two women who are at different amounts of risk for developing de novo stress urinary incontinence and need to make a similar decision before their surgery; should they have continence surgery or not at the time of surgery for pelvic organ prolapse. A surgeon will generally choose one of three approaches when discussing the risks of new-onset stress urinary incontinence and the potential benefits of performing a concomitant continence procedure:

(1) Treat all approach - In this approach, the surgeon communicates to the woman that she is 'at risk' of leaking after surgery. Several clinical trials recommend performing a continence procedure in all patients in this situation [3]. In one large trial, the average risk of urinary incontinence 1 year after surgery was $27 \%$ in women who have a concomitant midurethral sling placed and 43\% without a midurethral sling [3]. The advantage of this approach is that on average, a continence procedure performed during surgery for prolapse results in lower average rate of urinary incontinence compared with not performing a continence surgery. A disadvantage is that an average woman has a $57 \%$ chance that she will not leak even when continence surgery is not performed. If a surgeon routinely chooses this approach, on average, $57 \%$ of patients will receive an unnecessary procedure. The number of patients a surgeon needs to treat with a midurethral sling to prevent one case of urinary incontinence is 6.3. Another disadvantage is that the risk of adverse events increases with this approach. Rates of bladder perforation, urinary tract infection, major bleeding and incomplete bladder 
emptying were all significantly higher in the women receiving the continence surgery compared with sham [3].

(2) Wait and see approach - In contrast to a treat all approach, a surgeon may not perform the continence procedure at all and recommend performing the continence procedure only if the patient leaks after surgery (i.e. a staged approach). This option is less common as most patients prefer to have a single surgery rather than the possibility of having two surgeries and clinical trials have shown that, on average, there is a higher likelihood of improving incontinence symptoms using the first, treat all approach. An advantage of this approach is that a patient does not undergo a procedure they did not need (approximately 57\% will not need a sling). On average, this reduces the risk of adverse events in patients who did not need continence surgery as well as reduces cost. A disadvantage is approximately $40 \%$ of women will leak if no continence surgery is performed, and many of these patients will require a treatment for this incontinence resulting in increased distress after prolapse surgery.

(3) Crude estimate of risk approach - This is a common approach as it is consistent with combining evidence-based medicine and clinical judgment. The surgeon considers the presence of factors that may contribute to a patient's risk of leaking after surgery and counsels the patient accordingly. In theory, this approach should increase the rate of continence surgery in women at increased risk of leaking while lowering the rates of continence surgery in women at lower risk of leaking. Unfortunately, this approach makes multiple assumptions about the surgeon's existing knowledge of evidence and the systematic application of their clinical judgment in practice. It assumes that the surgeon knows and applies the important risk factors and their direction of effect (increase or decrease risk) to their decision. It also assumes that the caregiver assigns appropriate weight to each risk factor and can account for all risk factors together. In reality, humans in general are prone to many cognitive biases that make this method highly variable. Moreover, accounting for multiple risk factors such as age, BMI or diabetes is difficult and results in risk factors that are either ignored or not appropriately weighed in the decision process.

One attempt to standardize judgment is to use a mental algorithm, or heuristic. Female pelvic surgeons frequently use the presence or absence of leaking on a prolapse reduction urinary stress test or urodynamics as the sole predictor of whether to perform or not perform concomitant continence surgery. However, the predictive value of a prolapse reduction stress test is limited. The overall rate of postoperative stress urinary incontinence is approximately 50\% without a continence procedure and 16\% with a continence procedure in women without symptoms of stress urinary incontinence and a positive prolapse reduction stress test [4-8]. This $50 \%$ overall rate of postoperative stress urinary incontinence drops to $26 \%$ without a continence procedure and $17 \%$ with a continence procedure in women without symptoms of stress urinary incontinence and a negative prolapse reduction stress test.

(4) Prediction model approach - An alternative approach is to use a prediction model (http:// www.r-calc.com/ExistingFormulas.aspx?filter= CCQHS) to estimate a woman's individual risk $\left[9,10^{*}\right]$. In this case, information regarding clinical predictors is entered into an online calculator. Figures 1 and 2 illustrate the individual risks for the women presented in Cases 1 and 2.

In Case 1, despite a positive stress test, the woman's individual risk of leaking without a continence procedure is $28 \%$ and risk of leaking with a continence surgery is $6 \%$. The absolute reduction in risk is $22 \%$. However, for some women, a risk of $28 \%$ is already viewed as low for them to undergo a prophylactic procedure. This 'glass is half-full approach' by the woman is interpreted as a $72 \%$ chance they don't need a prophylactic procedure in the first place and this does not include the reduction in adverse events associated with performing a prophylactic continence surgery. In this case, the woman may choose not to have a prophylactic procedure.

Case 2 illustrates that despite a negative stress test, the woman's individual risk of leaking without a continence procedure is $63 \%$ and is $22 \%$ with a continence procedure. If a surgeon uses the stress test to triage a woman in this case into having or not having a continence procedure, this higher risk woman would not undergo a continence procedure. On the other hand, if the woman does have a concomitant continence surgery, she continues to have a 1-in-5 chance she will leak after surgery. The information the prediction model provides is important to convey when counseling the patient on what to expect after surgery. In both cases, the use of the model results in more accurate information being known about a woman's individual risk. Depending on the surgeon's treatment 


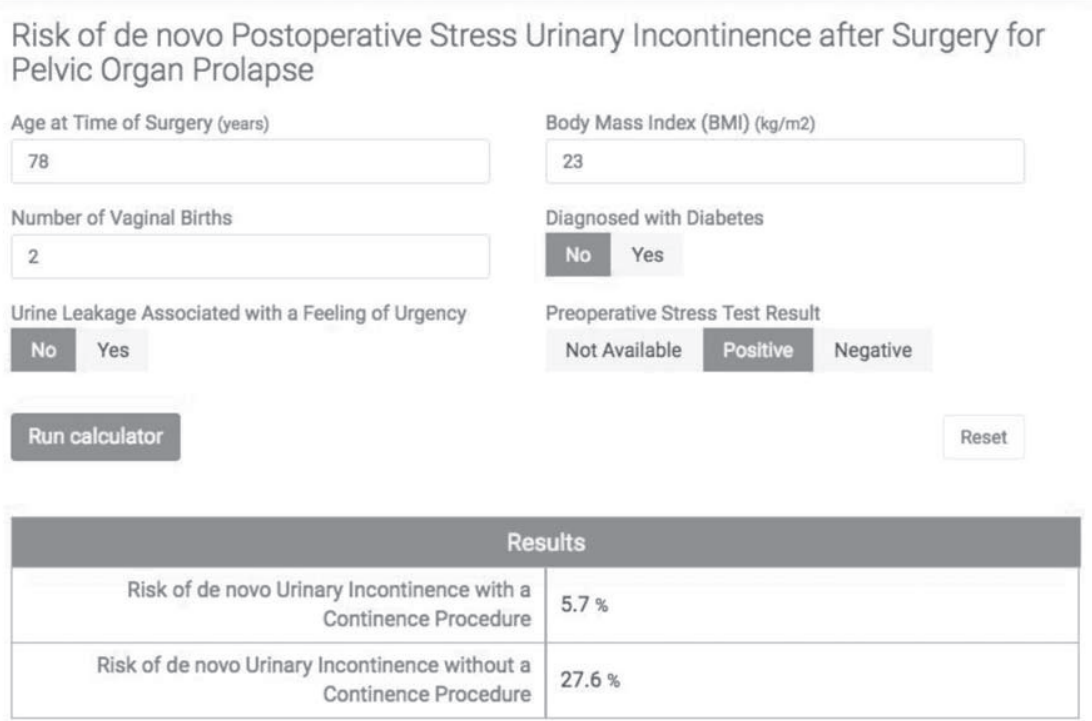

FIGURE 1. Online calculator illustrating an example of the individual risk of de novo stress urinary incontinence in a woman undergoing vaginal reconstructive surgery for pelvic organ prolapse. Despite the presence of leaking on a prolapse reduction stress test, the woman's risk is lower than that of the average woman.

algorithm, there is benefit to using this information during the decision-making process.

\section{MIXED URINARY INCONTINENCE AND PELVIC ORGAN PROLAPSE}

Case 3: The patient is a 78 -year-old para 2 woman who is planning to undergo a vaginal hysterectomy, uterosacral vaginal vault suspension, cystocele repair and cystoscopy for bothersome pelvic organ prolapse. She reports leaking small amounts of urine and urine leakage related to physical activity, coughing or sneezing that is moderately bothersome and sometimes leaking while trying to get to the bathroom. She reports intermittent difficulty emptying her bladder. She is active around the house and

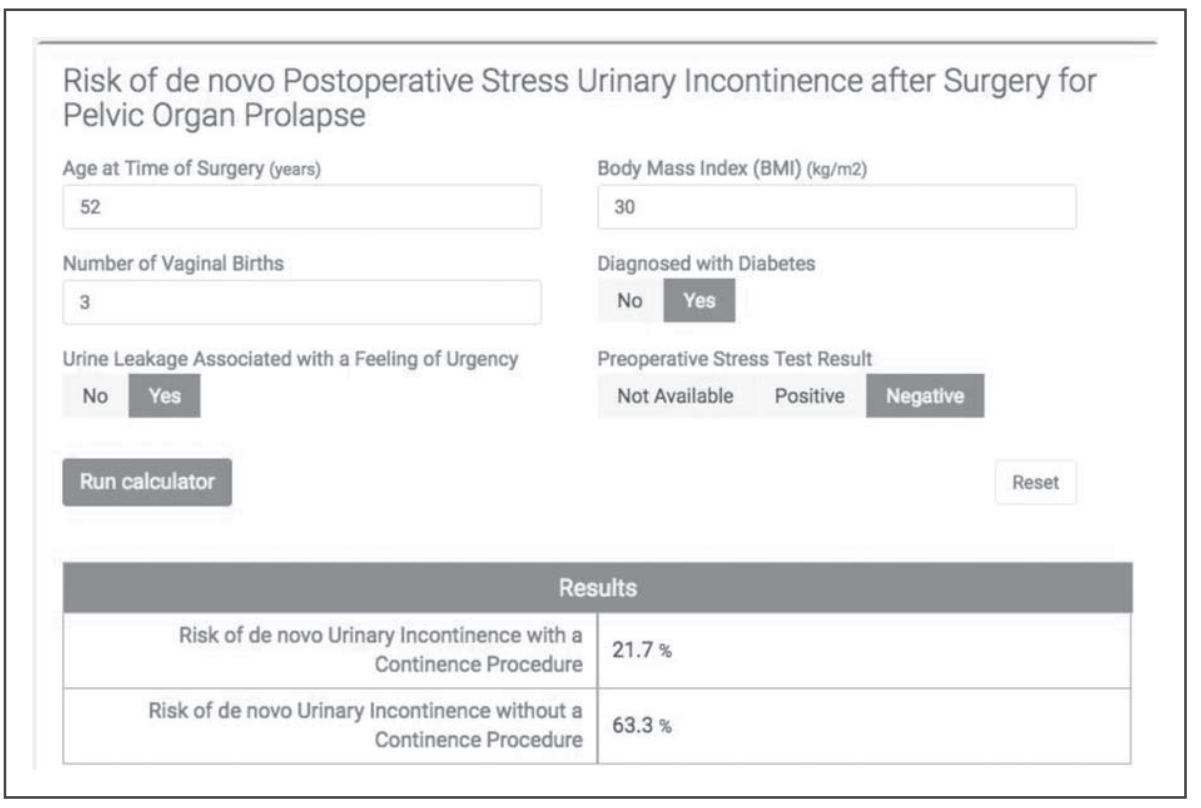

FIGURE 2. Online calculator illustrating an example of the individual risk of de novo stress urinary incontinence in a woman undergoing vaginal reconstructive surgery for pelvic organ prolapse. Despite the absence of leaking on a prolapse reduction stress test, the woman's risk is higher than that of the average woman. 
socially but reports that her ability to be physically active or go to events with her friends is affected by leaking urine. This causes frustration but she is not otherwise affected psychologically from her leaking. Her past medical history is significant for hypertension and osteoarthritis but no additional medical problems. On physical exam, her POPQ points are $\mathrm{Ba}+2$, C 0 and $\mathrm{Bp}-2$. Urodynamics were ordered, and her maximum uroflow rate is $10 \mathrm{ml} / \mathrm{s}$, maximum cystometric capacity is $400 \mathrm{ml}$ and lowest valsalva leak point pressure was $30 \mathrm{~cm}$ of water. She has decided to pursue surgical management of her pelvic organ prolapse, and the surgeon plans to perform a concomitant retropubic midurethral sling for her urinary incontinence. The surgeon must now discuss risks and benefits and of her risk of urinary incontinence after surgery.

It is common to offer women with stresspredominate mixed urinary incontinence symptoms treatment with a continence surgery during prolapse surgery. Surgical success rates in women with mixed urinary incontinence are typically less than success rates in women with pure stress urinary incontinence and surgeons are usually cautious in counseling women about the risks of worsening overactive bladder symptoms such as urgency, frequency or urgency urinary incontinence. Surgeons may tell the woman that, on average, evidence suggests that overactive bladder and incontinence symptoms improve after stress urinary incontinence surgery. A secondary analysis of several multicenter trials of women with mixed urinary incontinence determined that $50-70 \%$ of women reported reductions in overactive bladder and urge urinary incontinence symptoms [11]. However, compared with women with only stress urinary incontinence symptoms, women with mixed urinary incontinence symptoms have lower cure rates $[12,13]$.

There is currently no evidence-based optimal treatment strategy for surgically treating women with mixed urinary incontinence undergoing prolapse surgery. Therefore, the surgeon must carefully weigh the individual risks and benefits to the patient prior to proceeding with continence surgery. Unfortunately, crude estimates of risk are difficult to determine in women with mixed urinary incontinence. Additional risk factors such as age, BMI or diabetes are usually noted by surgeons but their effects on an individual patient's risk are difficult to determine. The risk of bothersome stress urinary incontinence for Case 3 is 31\%, bothersome urgency urinary incontinence is $46 \%$ and risk of any adverse event is $36 \%$ (Fig. 3). In this case, the model is helpful in providing information to the woman about her risk of having stress and urgency urinary incontinence after surgery.

\section{CLINICAL PREDICTION MODELS}

Predictive analytics continue to play key roles in improving the lives of humans. The modern day collision between 'Big Data' and new predictive analytics has resulted in personalization of everything from movie selections to healthcare recommendations.

In medicine, risk prediction models help clinicians personalize diagnostic and treatment information. They are useful in informing the multiple decisions that are made by care providers and their patients. In a diagnostic setting, these predictions are based on an estimated probability that a specific condition is present. In a prognostic setting, these predictions are based on whether an event will occur in the future. Practically, every domain of medicine is working toward creation, validation, updating and implementing prediction models [14"].

\section{MODEL DEVELOPMENT}

Prior to developing a prediction model, the first question one should ask is, 'will this model be used?' This leads to additional questions such as 'is the model going to be used in the setting in which it is intended?', 'will users of the model trust the predictors included in the model?' and 'are the predictors routinely available?' [15]

Model development begins with selecting predictors and combining them into a multivariable model. Choosing which candidate predictors to use is a critical step. Predictors should be easy to obtain in routine clinical practice, ideally have little variation in how they are defined and be available either before or at the time of the anticipated prediction. When choosing predictors, it is important to choose continuous variables over categorical variables when possible and it is generally not recommended to categorize linear variables into categorical variables (i.e. BMI) during data collection [16"'].

Choosing the type of model and the outcome are also important steps. The outcome should be clinically meaningful, well defined and acceptable for the community of users as an important outcome to predict. Logistic regression is commonly used when the outcome is a dichotomous (i.e. present or absent), and Cox regression is commonly used when long-term outcomes, such as time to the outcome, are important. However, the choice of the type of model should also include consideration of the best fit for the data in the model. For example, risk factors that are stable over time are very useful for a proportional hazards model such as Cox regression as this type of model assumes a constant hazard rate over time. On the other hand, if the risk 


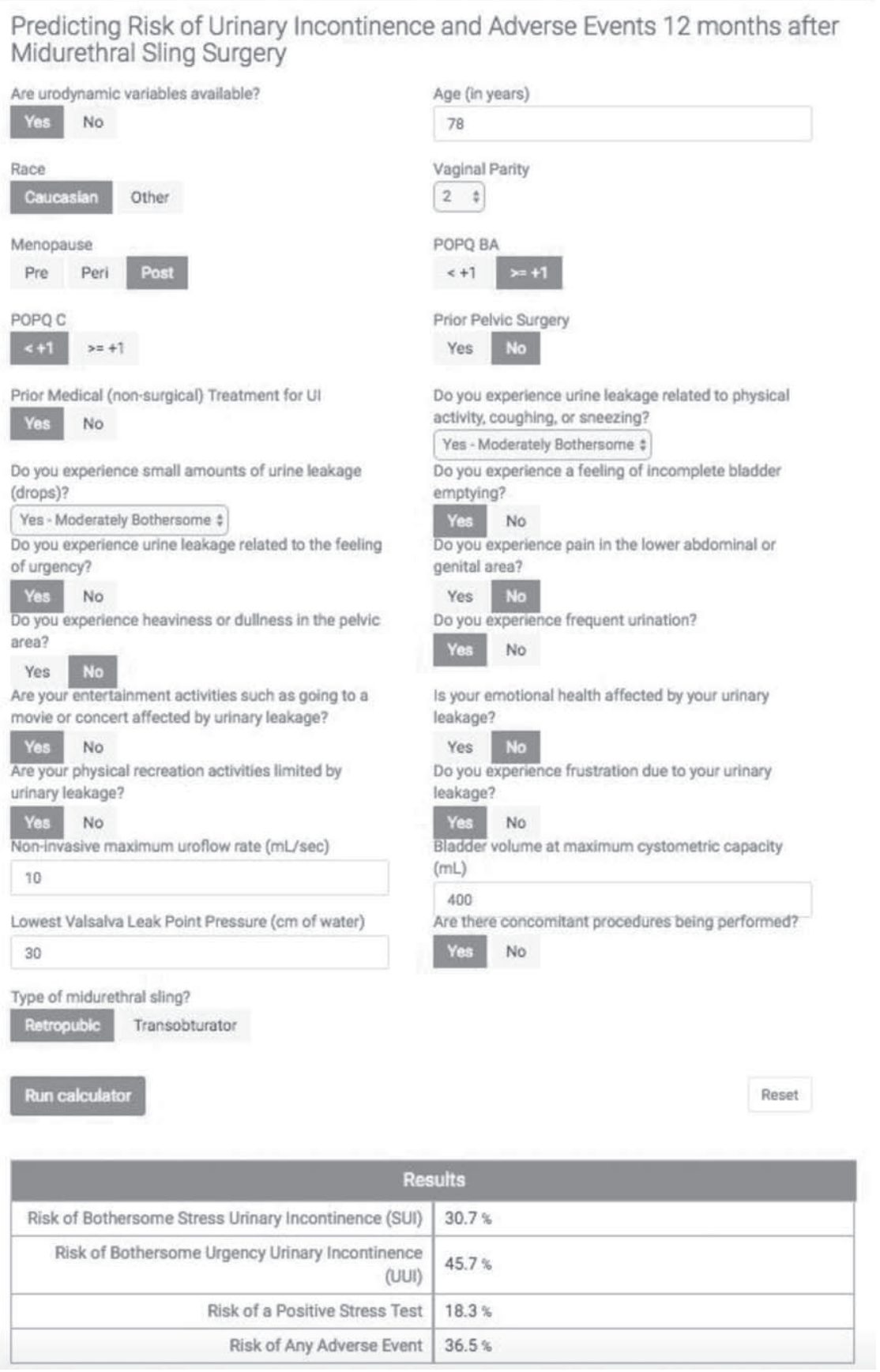

FIGURE 3. Online calculator illustrating an example of the individual risk of bothersome stress urinary incontinence, bothersome urgency urinary incontinence, a positive office urinary stress test and any adverse event in a woman undergoing vaginal reconstructive surgery for pelvic organ prolapse who reports stress-predominate mixed urinary incontinence symptoms.

factors wane over time, then a model that accounts for this variation, such as a parametric survival model using a log-normal distribution, may be considered [16"'].

There are multiple techniques for building prediction models including linear and logistic regression as well as tree-based methods such as regression and classification trees, bagging, boosting and random forests. The choice of which method starts with whether one believes there is a linear relationship between the predictors and the outcome. If so, then an approach using linear regression will likely work well and will outperform a method such as a regression tree that does not exploit this 
Table 1. Summary of models predicting individual risk of urinary incontinence after continence surgery

\begin{tabular}{|c|c|c|c|}
\hline \multirow[b]{2}{*}{ Outcome } & \multicolumn{2}{|c|}{ Internal validation } & \multirow{2}{*}{$\begin{array}{l}\text { External validation } \\
\text { Concordance index }\end{array}$} \\
\hline & Number of predictors & Concordance index & \\
\hline \multicolumn{4}{|c|}{ For women undergoing pelvic organ prolapse surgery without preoperative stress urinary incontinence } \\
\hline & & $N=457$ & $N=316$ \\
\hline Bothersome stress urinary incontinence ${ }^{a}$ & 7 & 0.73 & 0.62 \\
\hline \multicolumn{4}{|c|}{ For women undergoing midurethral sling surgery for stress urinary incontinence } \\
\hline & & $N=597$ & $N=902$ \\
\hline Bothersome stress urinary incontinence ${ }^{b}$ & 15 & 0.718 & 0.548 \\
\hline Stress test ${ }^{b}$ & 13 & 0.697 & 0.656 \\
\hline Bothersome urgency urinary incontinence ${ }^{b}$ & 12 & 0.697 & 0.621 \\
\hline Any adverse event ${ }^{b}$ & 11 & 0.636 & 0.567 \\
\hline
\end{tabular}

Table includes results previously reported by Jelovsek et al. $\left[9,10^{\text {"] }}\right.$.

aModel building and internal validation were performed using the Outcomes Following Vaginal Prolapse Repair and Midurethral Sling trial [20]. External validation was performed using the Colpopexy and Urinary Reduction Efforts trial [1].

${ }^{b}$ Model building and internal validation were performed using the Trial of midurethral slings dataset [21,22]. External validation was performed using a dataset from three combined clinical trials [23-26].

linear structure [17]. If one believes there is a highly nonlinear and complex association between the predictors and the outcome, then decision trees may outperform regression approaches.

\section{MODEL PERFORMANCE}

Once the model is built, it is important to assess the performance of the model in the setting in which it will be used. Model performance is typically measured by determining the model's discrimination and calibration. Discrimination is performed by ranking an individual's risk using a concordance index or $c$-statistic. Calibration is performed by observing whether the predicted probability was too high or low with a range of predicted probabilities using visual plots. Both measures are typically performed by assessing these properties on the same data from which the model was developed and using other participant data than was used for the model development [18]. Measuring a model's accuracy using data upon which the model was built results in an apparent performance of the model and frequently gives an overestimate (called overfitting) of its true performance. Therefore, it is necessary to perform internal validation to adjust the model for this overfitting. This results in a 'bias-corrected' concordance index. Internal validation techniques include cross-validation or bootstrapping [19]. External validation is performed by evaluating the model's performance in other participant data.

A summary of the discriminatory ability of the models used in the cases is presented in Table 1. The bias-corrected internal validation concordance index for the model predicting bothersome stress urinary incontinence 12 months after a woman undergoes pelvic organ prolapse surgery who does not have preoperative stress urinary incontinence is 0.73 [9]. This can be interpreted as the model was able to discriminate between high-risk and low-risk women $73 \%$ of the time. When this model's performance was tested on women undergoing sacral colpopexy for pelvic organ prolapse, the model's concordance index dropped to 0.62 . The model's performance was also compared against expert clinician's best guess and a preoperative urinary stress test. The prediction model outperformed expert clinicians (0.72 compared with $0.62, P<0.001)$ and preoperative urinary stress testing $(0.72 \mathrm{com}$ pared with $0.54, P<0.001$ ).

\section{CONCLUSION}

We have discussed how prediction models are helpful in several therapeutic decision-making contexts involving women planning to have surgery for stress urinary incontinence. Of course, prediction model development may be useful in a variety of additional pelvic-floor-related contexts. In prevention, models help identify women who may benefit from targeted interventions if they are at high risk. Prognostic models may help in counseling women by providing hope for recovery when a good prognosis is predicted and more aggressive treatment early in women with a low chance of benefit from first-line therapies. Overall, the aim of clinical prediction models is to help clinicians and women make better decisions with a model than without.

\section{Acknowledgements}

None. 


\section{Financial support and sponsorship}

The author has no financial, consultant, institutional or other relationships.

\section{Conflicts of interest}

There are no conflicts of interest.

\section{REFERENCES AND RECOMMENDED READING}

Papers of particular interest, published within the annual period of review, have been highlighted as

- of special interest

- of outstanding interest

1. Brubaker $L$, Cundiff $G W$, Fine $P$, et al. Abdominal sacrocolpopexy with Burch colposuspension to reduce urinary stress incontinence. N Engl J Med 2006; 354:1557-1566.

2. Ford AA, Rogerson L, Cody JD, Ogah J. Mid-urethral sling operations for stress urinary incontinence in women. Cochrane Database Syst Rev 2015; 7:CD006375.

3. Wei J, Nygaard I, Richter $\mathrm{H}$, et al. Outcomes following vaginal prolapse repair and mid urethral sling (OPUS) trial - design and methods. Clin Trials 2009; 6:162-171

4. Liang CC, Chang YL, Chang SD, et al. Pessary test to predict postoperative urinary incontinence in women undergoing hysterectomy for prolapse. Obstet Gynecol 2004; 104:795-800.

5. Visco AG, Brubaker L, Nygaard I, et al. The role of preoperative urodynamic testing in stress-continent women undergoing sacrocolpopexy: the Colpopexy and Urinary Reduction Efforts (CARE) randomized surgical trial. Int Urogynecol J Pelvic Floor Dysfunct 2008; 19:607-614.

6. Chaikin DC, Groutz A, Blaivas JG. Predicting the need for antiincontinence surgery in continent women undergoing repair of severe urogenital prolapse. J Urol 2000; 163:531-534

7. Gordon D, Gold RS, Pauzner D, et al. Combined genitourinary prolapse repair and prophylactic tension-free vaginal tape in women with severe prolapse and occult stress urinary incontinence: preliminary results. Urology 2001; 58:547-550.

8. Groutz A, Gold R, Pauzner D, et al. Tension-free vaginal tape (TVT) for the treatment of occult stress urinary incontinence in women undergoing prolapse repair: a prospective study of 100 consecutive cases. Neurourol Urodyn 2004; 23:632-635.

9. Jelovsek JE, Chagin $\mathrm{K}$, Brubaker L, et al. A model for predicting the risk of de novo stress urinary incontinence in women undergoing pelvic organ prolapse surgery. Obstet Gynecol 2014; 123 (2 Pt 1):279-287

10. Jelovsek JE, Hill AJ, Chagin KM, et al. Predicting risk of urinary incontinence

- and adverse events after midurethral sling surgery in women. Obstet Gynecol $2016 ; 127: 330-340$.

The aims of this study were to build and validate statistical models that could predict a woman's risk of developing bothersome stress urinary incontinence, bothersome urgency urinary incontinence, office stress test result and any adverse events 12 months after surgery. The authors used data from the Trial of MidUrethral Slings from the NIDDK Urinary Incontinence Treatment Network. All models were validated on data from four separate multicenter randomized trials. Models were able to discriminate between women who have high and low risk of events between 55 and $73 \%$ of the time and are available for public use using online calculators.
11. Zyczynski HM, Albo ME, Goldman $\mathrm{HB}$, et al. Change in overactive bladder symptoms after surgery for stress urinary incontinence in women. Obstet Gynecol 2015; 126:423-430.

12. Jain $P$, Jirschele $K$, Botros $S M$, Latthe $P M$. Effectiveness of midurethral slings in mixed urinary incontinence: a systematic review and meta-analysis. Int Urogynecol J 2011; 22:923-932

13. Gleason JL, Parden AM, Jauk V, et al. Outcomes of midurethral sling procedures in women with mixed urinary incontinence. Int Urogynecol J 2015; 26:715-720.

14. Collins GS, Reitsma JB, Altman DG, Moons KG. Transparent reporting of a

- multivariable prediction model for Individual Prognosis or Diagnosis (TRIPOD): the TRIPOD statement. J Clin Epidemiol 2015; 68:134-143.

Clinical predictions are routinely performed by healthcare providers on a daily basis. Unfortunately, these predictions rely on clinicians weighing multiple risk factors to derive a reliable estimate on an individual patient's actual risk. The last decade has seen an explosion in the number of published articles describing development and occasionally, the validation, of these models. The TRIPOD statement arose from a systematic review of the literature and expert consensus on the essence of good reporting of studies developing or validating multivariable prediction models. It is recommended that peer reviewers and editors use the 22item checklist when evaluating studies describing prediction model development and validation.

15. Reilly BM, Evans AT. Translating clinical research into clinical practice: impact of using prediction rules to make decisions. Ann Intern Med 2006; 144:201209.

16. Harrell F. Regression modeling strategies: with applications to linear models, - logistic regression, and survival analysis. New York: Springer; 2015.

This second edition is a must-read text for researchers interested in building and validating prediction models in medicine. The author, Frank Harrell, PhD, walks you through common problem-solving strategies faced when building multivariable prediction models in health care. The code and real data used in the text are publicly available for practice. He describes strategies for dealing with missing data, fitting models to nonlinear associations and how to handle situations in which too many variables exist in a dataset for appropriate analysis.

17. James G, Witten D, Hastie T, Tibshirani R. An introduction to statistical learning: with applications in R. New York: Springer; 2014

18. Pencina MJ, D'Agostino RBS. Evaluating discrimination of risk prediction models: the C statistic. JAMA 2015; 314:1063-1064.

19. Steyerberg EW, Harrell FE Jr, Borsboom GJJM, et al. Internal validation of predictive models: efficiency of some procedures for logistic regression analysis. J Clin Epidemiol 2001; 54:774-781.

20. Wei JT, Nygaard I, Richter HE, et al. A midurethral sling to reduce incontinence after vaginal prolapse repair. N Engl J Med 2012; 366:2358-2367.

21. Richter HE, Albo ME, Zyczynski HM, et al. Retropubic versus transobturator midurethral slings for stress incontinence. N Engl J Med 2010; 362:20662076.

22. Urinary Incontinence Treatment Network (UITN). The Trial of Mid-Urethral Slings (TOMUS): design and methodology. J Appl Res 2008; 8:AlboVol8No1.

23. Nager $\mathrm{CW}$, Brubaker $\mathrm{L}$, Litman $\mathrm{HJ}$, et al. A randomized trial of urodynamic testing before stress-incontinence surgery. N Engl J Med 2012; 366:19871997.

24. Nager $C W$, Brubaker $L$, Daneshgari $F$, et al. Design of the Value of Urodynamic Evaluation (ValUE) trial: a noninferiority randomized trial of preoperative urodynamic investigations. Contemp Clin Trials 2009; 30:531-539.

25. Barber MD, Kleeman S, Karram MM, et al. Transobturator tape compared with tension-free vaginal tape for the treatment of stress urinary incontinence: a randomized controlled trial. Obstet Gynecol 2008; 111:611-621.

26. Barber MD, Weidner AC, Sokol Al, et al. Single-incision mini-sling compared with tension-free vaginal tape for the treatment of stress urinary incontinence: a randomized controlled trial. Obstet Gynecol 2012; 119 (2 Pt 1):328-337. 